$(19,380$ characters $)$

\title{
cTAGE5 acts as a Sar1 GTPase regulator for collagen export
}

Norito Sasaki ${ }^{\mathrm{a}}$, Masano Shiraiwa ${ }^{\mathrm{a}}$, Miharu Maeda ${ }^{\mathrm{a}, \mathrm{b}}$, Tomohiro Yorimitsu ${ }^{\mathrm{c}}$, Ken Sato ${ }^{\mathrm{c}}$, Toshiaki Katada ${ }^{\mathrm{a}, \mathrm{d}}$, and Kota Saito ${ }^{\mathrm{a}, \mathrm{b}}$

aDepartment of Physiological Chemistry, Graduate School of Pharmaceutical Sciences, University of Tokyo, 7-3-1 Hongo, Bunkyo-ku, Tokyo 113-0033, Japan

${ }^{b}$ Department of Biological Informatics and Experimental Therapeutics, Graduate School of Medicine, Akita University 1-1-1, Hondo, Akita, 010-8543, Japan

${ }^{c}$ Department of Life Sciences, Graduate School of Arts and Sciences, University of Tokyo, Komaba, Meguro-ku, Tokyo, 153-8902, Japan

${ }^{\mathrm{d}}$ Faculty of Pharmacy, Musashino University, Tokyo, 202-8585, Japan

Running title: cTAGE5 as regulator for collagen export

Address correspondence to: Kota Saito (ksaito@med.akita-u.ac.jp) 


\section{ABSTRACT}

2 Secretory proteins synthesized within the endoplasmic reticulum (ER) are 3 exported via coat protein complex II (COPII)-coated vesicles. The formation of 4 the COPII-coated vesicles is initiated by activation of the small GTPase, Sar1.

5 cTAGE5 directly interacts with a guanine-nucleotide exchange factor (GEF), 6 Sec12, and a GTPase-activating protein (GAP) of Sar1, Sec23. We have 7 previously shown that cTAGE5 recruits Sec12 to the ER exit sites for efficient 8 production of activated Sar1 for collagen secretion. However, the functional 9 significance of the interaction between cTAGE5 and Sec23 has not been fully 10 elucidated. In this study, we showed that cTAGE5 enhances the GAP activity of 11 Sec23 toward Sar1. In addition, the interaction of cTAGE5 with Sec23 is 12 necessary for collagen exit from the ER. Our data suggests that cTAGE5 acts as a 13 Sar1 GTPase regulator for collagen secretion. 


\section{INTRODUCTION}

2 Secretory proteins synthesized within the endoplasmic reticulum (ER) are exported via

3 coat protein complex II (COPII)-coated vesicles (Miller and Schekman, 2013). COPII

4 vesicle formation is initiated by the activation of small GTPase, Sar1, by its

5 guanine-nucleotide exchange factor (GEF), Sec12 (Nakano and Muramatsu, 1989;

6 Barlowe and Schekman, 1993). The N-terminus amphipathic helix of activated Sar1

7 penetrates the ER membranes and induces membrane curvature (Lee et al., 2005; Long

8 et al., 2010; Settles et al., 2010). Sar1 then forms the pre-budding complex with

9 Sec23/Sec24, the inner coat complex. Sec24 interacts with cargo receptors to recruit

10 cargoes into nascent vesicles (Matsuoka et al., 1998; Bi et al., 2002; Sato and Nakano,

11 2005). Finally, Sec13/Sec31, the outer coat complex, enhances the GAP activity of

12 Sec23, inducing Sar1 GTP hydrolysis, and completes vesicle formation (Yoshihisa et

13 al., 1993; Antonny et al., 2001; Bi et al., 2007). Sec16, another peripheral membrane

14 protein, is also involved in COPII vesicle formation by modulating Sar1 GTPase

15 activity (Kung et al., 2012; Yorimitsu and Sato, 2012).

16 Mammalian cells export various cargoes, including bulky molecules, such as collagens

17 (Saito and Katada, 2015). It has been debated that how collagens, which form larger

18 than $300 \mathrm{~nm}$-long rigid structures are exported from the ER, since conventional

19 COPII-coated vesicles are 60-90 nm in diameter (Fromme and Schekman, 2005;

20 Malhotra and Erlmann, 2015). Recently, collagens were reported to be packaged into

21 COPII-coated large spherical structures (Gorur et al., 2017), and the mechanisms

22 underlying extra-sized COPII vesicle formation are emerging (Venditti et al., 2012;

23 Nogueira et al., 2014; Santos et al., 2015; Raote et al., 2017). We have identified

24 TANGO1 as a cargo receptor for collagens, and revealed that TANGO1 forms

25 membrane spanning macromolecular complex with multiple cTAGE5 and Sec12

26 molecules at ER exit sites (Saito et al., 2009; Maeda et al., 2016).

27 We previously reported that cTAGE5 recruits Sec12 to the ER exit sites (Saito et al.,

28 2014). Our data suggested that cTAGE5 does not affect GEF activity of Sec12 toward

29 Sar1 (Saito et al., 2014), but the concentration of Sec12 at ER exit sites accounts for

30 the efficient production of activated Sar1 required for collagen secretion (Tanabe et al.,

31 2016; Saito et al., 2017). On the contrary, we also reported that proline-rich domain

32 (PRD) of both cTAGE5 and TANGO1 interact with Sec23 and Sec24 by yeast

33 two-hybrid analysis (Saito et al., 2009; Saito et al., 2011), but the functional 
1 significance of these interactions has not been investigated.

2 In the present study, we revealed that cTAGE5 enhances the GAP activity of Sec23

3 towards Sar1. In addition, the interaction of cTAGE5 and Sec23 is required for

4 collagen exit from the ER. We proposed a model that cTAGE5 acts as a Sar1 GTPase

5 regulator for collagen secretion.

6

7 


\section{RESULTS AND DISCUSSION}

2 cTAGE5 enhances the GAP activity of Sec23/Sec24 toward Sar1

3 We have previously shown that cTAGE5 interacts with $\mathrm{Sec} 23 / \mathrm{Sec} 24$ (Saito et al., 4 2011); however, whether the interaction with cTAGE5 has any influence on the GAP 5 activity of Sec23 toward Sar1 has not yet been investigated. We measured the GTPase 6 activity of Sar1 by Pi release on the liposome in the presence of Sec23/Sec24 and 7 cTAGE5 (Figure S1). We purified the cytoplasmic domain of cTAGE5 (61-804 aa) 8 with $\mathrm{His}_{6}$ tag on the N-terminus, so that it can be recruited to DOGS-NTA-containing 9 liposome in the topology same as that of the physiological ER membranes (Cabrera et 10 al., 2014; Ebine et al., 2014). As shown in Figure 1A in columns 5 and 6, released Pi 11 due to the action of Sar1 GTPase was increased with the addition of Sec23/Sec24, 12 confirming that Sec23/Sec24 complex acts as a GAP for Sar1. Interestingly, when 13 His $_{6}$-tagged cTAGE5 cytoplasmic domain was introduced into the 14 DOGS-NTA-containing liposome, released Pi was significantly upregulated in the 15 presence of Sec23/Sec24 (Figure 1A, columns 6 and 9). However, cTAGE5 without $16 \mathrm{Sec} 23 / \mathrm{Sec} 24$ has no stimulating activity toward Sar1 GTPase (Figure 1A, columns 4, 5, 17 and 8), indicating that cTAGE5 does not act as a GAP by itself, but enhances the GAP 18 activity of Sec23 toward Sar1. Although the addition of $\mathrm{His}_{6}$-tagged Sec12 cytoplasmic 19 region (1-386 aa) alone has little effects on the production of free Pi by Sar1 (Figure 20 1A, column 5 and 7), the combination with Sec23/Sec24 significantly increased the 21 amount of released Pi (Figure 1A, column 11), consistent with the previous finding in 22 yeast (Barlowe and Schekman, 1993). Moreover, further addition of cTAGE5 23 increases the production of free Pi (Figure 1A, columns 11 and 12), confirming that 24 cTAGE5 enhances the GAP activity of Sec23 toward Sar1. Next, to check the 25 specificity of cTAGE5, we performed the assay with liposome free of DOGS-NTA, so 26 that the cTAGE5 is not attached to the liposome in this condition. As shown in Figure 27 1B, the addition of cTAGE5 failed to enhance the GAP activity of Sec23 in this 28 condition. In contrast, cTAGE5 efficiently enhances the GAP activity of Sec23 toward 29 Sar1 on the liposome with DOGS-NTA in a concentration-dependent manner (Figure 30 1C). As shown in Figure S2, the activity of cTAGE5 is statistically significant. 31 Interestingly, cTAGE5 enhances the GAP activity of Sec23 more efficiently than 32 Sec13/31 does (Please refer to the concentration of Figure 1C and D). It is probably 33 because cTAGE5 is attached to the membrane so that local concentration of cTAGE5 
1 is higher than that of Sec13/31 around membranes.

2

\section{Construction of cTAGE5 point mutants lacking Sec23A-binding activity}

4 We have previously shown that PRD of both cTAGE5 and TANGO1 interact with 5 Sec23/Sec24 by yeast two-hybrid assay (Saito et al., 2009; Saito et al., 2011). To 6 further analyze these interactions, we utilized recombinant proteins and checked 7 whether they directly interact by in vitro binding assay. Although PRD of both 8 cTAGE5 and TANGO1 efficiently interact with Sec23, they failed to bind Sec24 9 (Figure 2A). We concluded that cTAGE5 and TANGO1 directly bind to Sec23, but not 10 with Sec24. We then screened cTAGE5 mutant incapable of binding to Sec23. We employed mutations in cTAGE5 PRD by error-prone PCRs and isolated clones, which 12 exhibited reduced interaction with $\operatorname{Sec} 23$ by yeast two-hybrid assay. Most of the 13 positive clones contained non-sense mutations; however, we identified one missense mutation, R757G (Figure 2B and C).

15 During our analysis, Ma et al. reported that PPP motifs in the PRD regions of both cTAGE5 and TANGO1 are responsible for the interaction with Sec23A (Ma and 17 Goldberg, 2016). Thus, we also prepared 4PA mutant, in which four regions of 18 consecutive prolines were changed to alanines (a total of 15 prolines were changed to 19 alanines) (Ma and Goldberg, 2016) (Figure 2C). As shown in Figure 2B, the 4PA 20 mutant was also incapable of interacting with Sec23 in the yeast two-hybrid assay. 21 Next, we checked the interaction by in vitro binding assay. Both RG and 4PA mutants 22 showed reduced binding to $\operatorname{Sec} 23$, consistent with the yeast two-hybrid assay (Figure $232 \mathrm{D}$ and $\mathrm{E})$.

24 Then, we examined whether mutants correctly localized to the ER exit sites. As 25 shown in Figure 2F, both RG and 4PA mutants extensively co-localized with Sec16, a 26 bone-fide ER exit site marker, suggesting that the mutations do not affect its 27 localization. We previously reported that the localization of the ER exit sites within the 28 ER is defined by the interaction between TANGO1 and Sec16, and cTAGE5 is 29 recruited to the ER exit sites by the interaction with TANGO1 (Maeda et al., 2017). 30 Current data further supported the idea that cTAGE5 is not likely to be localized to the 31 ER exit sites by the interaction with Sec23/Sec24.

32 Next, we checked whether cTAGE5 mutants retain the properties to interact with 33 TANGO1 and Sec12. As shown in Figure $2 \mathrm{G}$ and $\mathrm{H}$, both the mutants were still bound 
1 to TANGO1L and Sec12, indicating that the mutations do not destroy the overall

2 conformation of cTAGE5, except for its affinity to Sec23.

3

4 PRD of cTAGE5 is responsible for enhancing the GAP activity of Sec23

Because PRD of cTAGE5 is responsible for interacting with Sec23, we speculated that this domain is also involved in the GAP enhancing activity against Sec23. Thus, we made recombinant PRD of cTAGE5 and checked the activity against Sec23. As shown in Figures 3 and S3, the addition of cTAGE5 PRD into DOGS-NTA liposome with Sar1, Sec12, and Sec23/Sec24 significantly enhanced the Pi release in a concentration-dependent manner. Conversely, the addition of GST has no effects on the free Pi production (Figures 3 and S3), indicating that cTAGE5 PRD has a property to enhance the GAP activity of Sec23 toward Sar1. Next, we examined the effects of cTAGE5 mutants with reduced Sec23-binding on the GAP enhancing activity. Interestingly, although RG mutant retained the activity at the normal level, 4PA mutant failed to enhance the GAP activity of Sec23 toward Sar1 (Figures 3 and S3). These results indicated that the domain responsible for GAP-enhancing activity and Sec23-binding could be separable within the PRD of cTAGE5.

\section{cTAGE5 with reduced Sec23-binding activity failed to secrete collagen VII from} the ER

Next, we examined whether the mutants could promote collagen VII secretion from the ER. As previously reported (Saito et al., 2014; Maeda et al., 2016; Tanabe et al., 2016), we quantified the signals of accumulated collagen VII within the ER as an index of its secretion. The expression of both the mutants with reduced Sec23-binding activity to the ER exit sites could not rescue the block of collagen VII secretion induced by cTAGE5 knockdown, although the wild-type cTAGE5 rescued collagen secretion (Figure 4).

\section{cTAGE5 acts as a Sar1 GTPase regulator for collagen export}

cTAGE5 is an integral membrane protein containing two coiled coil domains and PRD in the cytoplasmic region. cTAGE5 belongs to the cTAGE gene family, consisting of 6 genes coding for highly homologous proteins and 9 pseudogenes according to the HUGO Gene Nomenclature Committee (Comtesse et al., 2001). Until 
1 now, we have been focusing on studying cTAGE5, and the possibility that the

2 antibodies we used might cross-react with other cTAGE-family proteins cannot be

3 ruled out. However, this seems unlikely because the siRNAs that we used efficiently

4 depleted proteins recognized by cTAGE5 antibodies, and we revealed these siRNAs

5 are specific for cTAGE5, and not cross-reactive with the other five proteins, by 6 analyzing RefSeq database (Saito et al., 2011; Saito et al., 2014; Tanabe et al., 2016).

7 Thus, we believe that our study describes the results only for cTAGE5, and the

8 influence on other cTAGE family members would be very limited. Recently, cTAGE5

9 has been reported to be involved in collagen, VLDL, and insulin secretion (Saito et al.,

10 2011; Saito et al., 2014; Santos et al., 2016; Tanabe et al., 2016; Wang et al., 2016;

11 Fan et al., 2017). We previously reported that cTAGE5 directly interacts with Sec12

12 via cytoplasmic region just after the membrane-spanning domain and this interaction is

13 specifically required for collagen secretion (Saito et al., 2014; Tanabe et al., 2016). In

14 addition, we revealed that the second coiled coil domain of cTAGE5 is responsible for

15 interacting with TANGO1, a collagen cargo receptor (Saito et al., 2011), and cTAGE5

16 can form homo-multimer (Maeda et al., 2016).

17 In this study, we revealed that the PRD of cTAGE5 is not only responsible for Sec23

18 binding, but also for the activation of Sec23 GAP activity toward Sar1. Thus, we

19 proposed that TANGO1 recruits cTAGE5 multimer for regulation of Sar1 GTPase in

20 the vicinity of the ER exit sites. Sec12, concentrated around ER exit sites via

21 interaction with cTAGE5, efficiently produces the activated Sar1 around ER exit sites.

22 Sar1, then, might be involved in the collagen-containing tubule formation (See the

23 discussion blow), and is efficiently hydrolyzed by Sec23, the activity of which is

24 enhanced by the interaction with cTAGE5 or Sec31. This hydrolysis of GTP by Sar1

25 might be important for completing the collagen-containing carrier formation.

26 Notably, in the in vitro assay, liposomes incubated with the GTP-restricted form of 27 Sar1 mutant (Sar1 H79G) or with Sar1 and non-hydrolyzable GTP analogs, such as 28 GMP-PNP and GTP $\gamma$ S, induced tubules long enough to accommodate collagens inside 29 (Long et al., 2010; Bacia et al., 2011). These tubular structures still attach to the 30 liposome, implying that GTP hydrolysis by Sar1 is necessary for carriers to detach 31 from liposomes. Moreover, cryo-electron microscopy analysis revealed that giant 32 unilamellar vesicles incubated with non-hydrolyzable Sar1, Sec23/Sec24, and 33 Sec13/Sec31 produce tubes coated with $\operatorname{Sec} 23 / \operatorname{Sec} 24$ and Sec13/Sec31. Interestingly, 
1 the predicted model indicates that tubule coated with $\operatorname{Sec} 23 / \mathrm{Sec} 24$ recruits less

2 Sec13/Sec31 than the spherical vesicles do (Zanetti et al., 2013). Thus, it is interesting

3 to speculate that cTAGE5-mediated activation of Sec23 GAP activity might be

4 important only for completing the formation of large cargo carriers from the tubular 5 structures.

6 On the contrary, Sec31 has also been reported to be involved in collagen secretion.

7 Ubiquitylation of Sec31 regulated by calcium-binding proteins and Cul3-KLHL12

8 leads to the large carrier formation (Jin et al., 2012; McGourty et al., 2016). It has not

9 been investigated whether ubiquitylation of $\operatorname{Sec} 31$ has any effects on the activity

10 toward Sec23. The mechanism underlying the coordination between the

11 GAP-enhancing activities of Sec31 and cTAGE5 awaits further investigation.

12 Interestingly, RG mutant, which retains the property to enhance the Sec23 GAP

13 activity, but exhibits reduced binding to Sec23, fails to secrete collagen VII from the

14 ER. These data imply that the regions of cTAGE5 responsible for GAP enhancing 15 activity and interaction with Sec23 could be separable within the cTAGE5 PRD. 16 Alternatively, if the same regions are responsible for the activity and interaction, it 17 suggests that RG mutant has a higher GAP enhancing activity than that of wild-type 18 cTAGE5. It also suggested that interaction of cTAGE5 with Sec23 is necessary for 19 collagen secretion, in addition to enhancing the GAP activity of Sec23. How this 20 interaction participates in collagen secretion needs to be revealed in the future studies.

21 In this study, we revealed that cTAGE5 enhances the GAP activity of Sec23 toward 22 Sar1. In addition, the interaction of cTAGE5 and Sec23 is necessary for collagen exit 23 from the ER. Thus, cTAGE5 acts as a Sar1 GTPase regulator for collagen secretion. 


\section{MATERIALS AND METHODS}

\section{Antibodies}

3 Anti-collagen VII monoclonal antibody (NP-185) was kindly provided by Dr. Lynn

4 Sakai. Other antibodies were used as described previously (Maeda et al., 2017).

\section{Constructs}

7 cTAGE5 rescue constructs were made as described previously (Saito et al., 2014;

8 Maeda et al., 2017). cTAGE5-4PA construct was made by introducing following 9 mutations (P693A P694A P695A P720A P721A P722A P723A P724A P743A P744A 10 P745A P771A P772A P773A P774A).

\section{Cell culture and transfection}

13 HeLa, HSC-1, and 293T cells were cultured in DMEM supplemented with $10 \%$ fetal 14 bovine serum. Lipofectamine RNAi max (Thermofisher) was used for transfecting 15 siRNA. For plasmids transfection, polyethylenimine "MAX" (polysciences) or 16 FuGENE 6 (Promega) were used.

\section{Recombinant human protein purification}

19 Proteins used for GTPase hydrolysis assay were all from humans. Baculovirus 20 encoding FLAG-Sec12 (1-386 aa)-His ${ }_{6}$, His $_{6}$-cTAGE5 (61-804 aa)-FLAG was made 21 with Bac-to-Bac Baculovirus Expression System according to manufacturer's protocol 22 (Life Technologies). Sf9 cells infected with virus were collected. Each protein was 23 purified with FLAG M2 agarose beads (Sigma-Aldrich). Elution was made with FLAG 24 peptide, then the buffers were exchanged with $20 \mathrm{mM}$ HEPES-KOH (pH 7.4), $160 \mathrm{mM}$ $25 \mathrm{KOAc}, 1 \mathrm{mM} \mathrm{MgCl}$ by desalting column. Sec13/FLAG-Sec31a was cloned into 26 pFastBacDual vector and baculovirus was produced. Protein was purified from 27 infected Sf9 cells with FLAG M2 agarose beads (Sigma-Aldrich) followed by elution 28 with FLAG peptide. The buffer was exchanged with TBS/5\% (w/v) glycerol by 29 desalting column. FLAG-Sec23A, FLAG-Sec24D were expressed in 293T cells and 30 purified with FLAG M2 agarose beads (Sigma-Aldrich). Elution was made with FLAG 31 peptide. The buffer was exchanged with TBS/0.05\% Lubrol-PX by desalting column. 32 HA-Sec23/FLAG-Sec24 were expressed in 293T cells and purified with FLAG M2 33 agarose beads (Sigma-Aldrich). Elution was made with FLAG peptide. The buffer was 
1 exchanged with TBS/5\% (w/v) glycerol by desalting column. GST-Sar1a was 2 expressed in Escherichia coli and purified with glutathione sepharose (GE Healthcare).

3 Then GST tag was cleaved by thrombin protease followed by dialysis with $20 \mathrm{mM}$ 4 HEPES-KOH ( $\mathrm{pH}$ 6.8), $160 \mathrm{mM} \mathrm{KOAc,} 5 \mathrm{mM} \mathrm{MgCl}_{2}, 5 \mathrm{mM} \beta$-ME, $0.5 \mathrm{mM}$ AEBSF, $510 \mu \mathrm{M}$ GDP, 5\% (w/v) glycerol. His ${ }_{6}$-cTAGE5 (651-804 aa)-GST wild-type, 6 His $_{6}$-cTAGE5 (651-804 aa)-GST R757G, His 6 -cTAGE5 (651-804 aa)-GST 4PA were 7 expressed in Escherichia coli and purified with glutathione sepharose (GE Healthcare). 8 The GST tags were cleaved by prescission protease and further purified with $\mathrm{Ni}$ 9 sepharose 6 Fast Flow (GE Healthcare) and eluted with imidazole. The buffers were 10 exchanged with TBS by desalting columns. His ${ }_{6}$-GST expressed in Escherichia was 11 purified by Ni sepharose 6 Fast Flow and eluted with imidazole. The buffer was 12 exchanged with TBS by desalting column.

\section{Liposome Preparation}

15 Lipids were purchased from Avanti Polar Lipids, except sphingomyelin (Enzo life 16 science) and cholesterol (nakalai tesque). The lipid mixture was evaporated and then 17 resuspended in $20 \mathrm{mM}$ Hepes-KOH, $\mathrm{pH} 7.4,160 \mathrm{mM} \mathrm{KOAc}, 1 \mathrm{mM} \mathrm{MgCl}$ followed 18 by sonication. Liposome without $\mathrm{Ni}$ consists of di-oleoyl-phosphatidylcholine (DOPC; 19 54\%), di-oleoyl-phosphatidyl-ethanolamine (DOPE; 21\%), soy phosphatidylinositol 20 (PI; 9\%), cholesterol (Cho; 7\%), di-oleoyl-phosphatidyl-serine (DOPS; 3\%), 21 sphingomyelin (SM; 3\%), cystidine diphosphate diacylglycerol (CDP-DAG; 2\%), 22 dioleoyl-phosphatidic acid (DOPA; 1\%). Liposome with Ni (20\%) consists of DOPC; $2341 \%$, DOPE; 18\%, PI; 5\%, Cho; 7\%, DOPS; 3\%, SM; 3\%, CDP-DAG; 2\%, DOPA; $241 \%$, and 1,2-Dioleoyl-sn-Glycero-3-[(N-(5-amino-1-carboxypentyl)iminodiacetic 25 acid)succinyl](nickel salt)(DGS-NTA; 20\%). Liposome with Ni (10\%) consists of 26 DOPC; 48.6\%, DOPE; 18.9\%, PI; 8.1\%, Cho; 6.3\%, DOPS; 2.7\%, SM; $2.7 \%$, 27 CDP-DAG; $1.8 \%$, DOPA; $0.9 \%$, and DGS-NTA; $10 \%$.

\section{GTP hydrolysis assay}

30 His $_{6}$-cTAGE5 (61-804 aa)-FLAG, FLAG-Sec12 (1-386 aa)-His ${ }_{6}$ and liposome were 31 preincubated in a buffer consisting of $20 \mathrm{mM}$ Hepes-KOH, $\mathrm{pH}$ 7.4, $160 \mathrm{mM} \mathrm{KOAc}, 1$ $32 \mathrm{mM} \mathrm{MgCl}_{2}$ for $2 \mathrm{~h}$ at $4^{\circ} \mathrm{C}$. Then, Sar1 GTP hydrolysis were initiated in a buffer 33 consisting of $25 \mu \mathrm{g} / \mathrm{ml}$ of liposome, $20 \mathrm{mM}$ HEPES-KOH (pH 7.4), $100 \mathrm{mM} \mathrm{KOAc,} 6$ 
$\mathrm{mM}$ Tris- $\mathrm{HCl}, 45 \mathrm{mM} \mathrm{NaCl}, 1 \mathrm{mM} \mathrm{MgCl} 2,1.6 \%$ Glycerol, $133 \mathrm{nM}$ GDP, $2 \mu \mathrm{M}\left[\gamma{ }^{32} \mathrm{P}\right]$

GTP, $50 \mu \mathrm{M}$ AppNHp, $67 \mu \mathrm{M} \beta$-ME, $67 \mu \mathrm{M}$ AEBSF, in the presence or absence of 8 nM FLAG-Sec12 (1-386 aa)-His 6 , 15 nM HA-Sec23A/FLAG-Sec24D, and $200 \mathrm{nM}$ Sar1, and indicated concentrations of His $_{6}$-cTAGE5 (61-804 aa)-FLAG and Sec13/31. His $_{6}$-GST or His - -cTAGE5 (651-804 aa)-GST wild-type or R757G or 4PA, FLAG-Sec12 (1-386 aa)-His ${ }_{6}$ and liposome were preincubated in a buffer consisting of $20 \mathrm{mM}$ Hepes-KOH, pH 7.4, $160 \mathrm{mM} \mathrm{KOAc,} 1 \mathrm{mM} \mathrm{MgCl}$ for $2 \mathrm{~h}$ at $4^{\circ} \mathrm{C}$. Then, Sar 1

8 GTP hydrolysis were initiated in a buffer consisting of $25 \mu \mathrm{g} / \mathrm{ml}$ of liposome, $20 \mathrm{mM}$ HEPES-KOH (pH 7.4), $100 \mathrm{mM} \mathrm{KOAc,} 8 \mathrm{mM}$ Tris-HCl, $60 \mathrm{mM} \mathrm{NaCl}, 1 \mathrm{mM} \mathrm{MgCl}$, 1.6\% Glycerol, $133 \mathrm{nM}$ GDP, $2 \mu \mathrm{M}\left[\gamma^{-32} \mathrm{P}\right] \mathrm{GTP}, 50 \mu \mathrm{M}$ AppNHp, $67 \mu \mathrm{M} \beta-\mathrm{ME}, 67$ $1 \mu \mathrm{M}$ AEBSF, 8 nM FLAG-Sec12 (1-386 aa)-His ${ }_{6}, 15$ nM HA-Sec23A/FLAG-Sec24D, 2 and $200 \mathrm{nM}$ Sar1, and indicated concentrations of $\mathrm{His}_{6}$-GST, His ${ }_{6}$-cTAGE5 (651-804 aa)-GST wild-type, R757G, 4PA. The reaction mixtures were incubated for $1 \mathrm{~h}$ at $430^{\circ} \mathrm{C}$, and quenched by adding $750 \mu \mathrm{L}$ of ice-cold $5 \%(w / v)$ Norit SX-Plus (Wako, Japan) in $50 \mathrm{mM} \mathrm{NaH}_{2} \mathrm{PO}_{4}$, then centrifuged at $9,000 \mathrm{~g}$ for $15 \mathrm{~min}$ at $4^{\circ} \mathrm{C} .300 \mu \mathrm{L}$ of 6 supernatants were mixed with $1 \mathrm{~mL}$ of Clear-sol I (Nacalai-tesque, Japan) and free ${ }^{32} \mathrm{Pi}$ 7 was measured.

\section{Random mutagenesis screening}

0 Random mutagenesis was essentially performed as described previously (Cadwell and 1 Joyce, 1992). Error-prone PCR was performed with Titanium Taq polymerase (Takara, Japan) in the presence of $0.64 \mathrm{mM} \mathrm{MnCl}_{2}$ with unbalanced ratio of nucleotides $(0.2$ $3 \mathrm{mM}$ dATP, $1 \mathrm{mM}$ dTTP, $1 \mathrm{mM}$ dGTP, $1 \mathrm{mM}$ dCTP). PCR products were cloned into 4 pGADT7 for following yeast two-hybris analysis. AH109 yeast strain was transformed 5 with pGBKT7 and pGADT7 vectors and plated on tryptophan- and leucine-deficient 6 plate. The colonies were re-plated onto tryptophan-, leucine-, histidine-, and 7 adenine-deficient plate. The colonies, which failed to grow on the tryptophan-, leucine-, 8 histidine-, and adenine-deficient plate were picked and lysed by zymolyase for 9 sequence analysis.

2 GST-cTAGE5 (651-804 aa)-His ${ }_{6}$ wild-type, GST-cTAGE5 (651-804 aa)-His ${ }_{6}$ R757G, 33 GST-cTAGE5 (651-804 aa)-His ${ }_{6} 4 \mathrm{PA}$ expressed in Escherichia were purified with 
1 glutathione sepharose followed by elution with glutathione. Eluates were then purified

2 with Ni sepharose 6 Fast Flow and eluted with imidazole. The buffers were exchanged 3 with TBS/0.05\% Lubrol-PX by desalting columns. In vitro binding assay was 4 essentially performed as described previously (Maeda et al., 2017). In brief, GST, 5 GST-tagged cTAGE5-PRD (651-804 aa)-His ${ }_{6}$ constructs were conjugated to 6 glutathione sepharose and incubated with FLAG-Sec23A. Beads were washed with $7 \quad$ TBS/0.05\% Lubrol-PX for five times followed by elution with glutathione.

\section{$9 \quad$ Immunoprecipitation and Western blotting}

10 The experiments were performed essentially as described previously (Maeda et al., 11 2017). In brief, Cells extracted with extraction buffer (20 mM Tris- $\mathrm{HCl}$ (pH 7.4), 100 $12 \mathrm{mM} \mathrm{NaCl}, 1 \mathrm{mM}$ EDTA, $1 \%$ Triton X-100, and protease inhibitors) were centrifuged 13 at $100,000 \times g$ for $30 \mathrm{~min}$ at $4^{\circ} \mathrm{C}$. Cell lysates were immunoprecipitated with FLAG 14 M2 Agarose beads (Sigma-Aldrich). The beads were washed five times with $15 \mathrm{TBS} / 0.1 \%$ Triton $\mathrm{X}-100$ and processed for sample preparation.

\section{Immunofluorescence microscopy}

18 Immunofluorescence microscopy analysis was performed as described previously 19 (Maeda et al., 2017). Cells grown on coverslips were washed with phosphate-buffered 20 saline (PBS), fixed with methanol $\left(6 \mathrm{~min}\right.$ at $\left.-20^{\circ} \mathrm{C}\right)$, and then washed with PBS and 21 blocked in blocking solution (5\% bovine serum albumin in PBS with $0.1 \%$ Triton $22 \mathrm{X}-100$ for $15 \mathrm{~min}$ ). After blocking, cells were stained with primary antibody for $1 \mathrm{~h}$, 23 followed by incubation with Alexa Fluor-conjugated secondary antibodies for $1 \mathrm{~h}$ at 24 room temperature. Images were acquired with confocal laser scanning microscopy 25 (LSM700; Plan-Apochromat 63×/1.40 numerical aperture [NA] oil immersion 26 objective lens; Carl Zeiss, Oberkochen, Germany). The acquired images were 27 processed with Zen 2009 software (Carl Zeiss). All imaging was performed at room 28 temperature.

\section{Quantification of collagen VII staining}

31 Quantification of collagen VII accumulation was essentially performed as described 32 previously (Tanabe et al., 2016). Stained cells were analyzed by Zeiss Axio Imager M1 33 microscopy (EC Plan-Neofluar 40×/ 0.75 NA objective lens) and processed with 
1 AxioVision software (Carl Zeiss). Area calculation and intensity scanning were done 2 by ImageJ software (National Institutes of Health, Bethesda, MD).

3

4 Acknowledgements

5 We thank Dr. Lynn Sakai for providing us NP185 antibody. We also thank members of

6 the Katada lab for valuable discussions. This work was supported in part by research

7 grants from Japan Society for the Promotion of Science (JSPS) (grant numbers

8 17J07885 to M.M.; 23229001 to T.K., 26440046, 17H03651 to K.S.).

9 


\section{REFERENCES}

2 Antonny, B., Madden, D., Hamamoto, S., Orci, L., and Schekman, R. (2001).

3 Dynamics of the COPII coat with GTP and stable analogues. Nat. Cell Biol. 3, $4 \quad 531-537$.

5 Bacia, K., Futai, E., Prinz, S., Meister, A., Daum, S., Glatte, D., Briggs, J.A., and

6 Schekman, R. (2011). Multibudded tubules formed by COPII on artificial liposomes. 7 Sci. Rep. $1,17$.

8 Barlowe, C., and Schekman, R. (1993). SEC12 encodes a

9 guanine-nucleotide-exchange factor essential for transport vesicle budding from the 10 ER. Nature 365, 347-349.

11 Bi, X., Corpina, R.A., and Goldberg, J. (2002). Structure of the Sec23/24-Sar1

12 pre-budding complex of the COPII vesicle coat. Nature 419, 271-277.

13 Bi, X., Mancias, J.D., and Goldberg, J. (2007). Insights into COPII coat nucleation 14 from the structure of Sec23.Sar1 complexed with the active fragment of Sec31. Dev. 15 Cell 13,635-645.

16 Cabrera, M., Nordmann, M., Perz, A., Schmedt, D., Gerondopoulos, A., Barr, F., 17 Piehler, J., Engelbrecht-Vandre, S., and Ungermann, C. (2014). The Mon1-Ccz1 GEF 18 activates the Rab7 GTPase Ypt7 via a longin-fold-Rab interface and association with 19 PI3P-positive membranes. J. Cell Sci. 127, 1043-1051.

20 Cadwell, R.C., and Joyce, G.F. (1992). Randomization of genes by PCR mutagenesis. 21 PCR Methods Appl. 2, 28-33.

22 Comtesse, N., Reus, K., and Meese, E. (2001). The MGEA6 multigene family has an 23 active locus on $14 \mathrm{q}$ and at least nine pseudogenes on different chromosomes. 24 Genomics 75, 43-48.

25 Ebine, K., Inoue, T., Ito, J., Ito, E., Uemura, T., Goh, T., Abe, H., Sato, K., Nakano, A., 26 and Ueda, T. (2014). Plant vacuolar trafficking occurs through distinctly regulated 27 pathways. Curr. Biol. 24, 1375-1382.

28 Fan, J., Wang, Y., Liu, L., Zhang, H., Zhang, F., Shi, L., Yu, M., Gao, F., and Xu, Z. 29 (2017). cTAGE5 deletion in pancreatic beta cells impairs proinsulin trafficking and 30 insulin biogenesis in mice. J. Cell Biol.

31 Fromme, J.C., and Schekman, R. (2005). COPII-coated vesicles: flexible enough for 32 large cargo? Curr. Opin. Cell Biol. 17, 345-352.

33 Gorur, A., Yuan, L., Kenny, S.J., Baba, S., Xu, K., and Schekman, R. (2017). 
1 COPII-coated membranes function as transport carriers of intracellular procollagen I. J.

2 Cell Biol. 216, 1745-1759.

3 Jin, L., Pahuja, K.B., Wickliffe, K.E., Gorur, A., Baumgartel, C., Schekman, R., and

4 Rape, M. (2012). Ubiquitin-dependent regulation of COPII coat size and function.

5 Nature 482, 495-500.

6 Kung, L.F., Pagant, S., Futai, E., D'Arcangelo, J.G., Buchanan, R., Dittmar, J.C., Reid,

7 R.J., Rothstein, R., Hamamoto, S., Snapp, E.L., Schekman, R., and Miller, E.A. (2012).

8 Sec24p and Sec16p cooperate to regulate the GTP cycle of the COPII coat. EMBO J.

$9 \quad 31,1014-1027$.

10 Lee, M.C., Orci, L., Hamamoto, S., Futai, E., Ravazzola, M., and Schekman, R. (2005).

11 Sar1p N-terminal helix initiates membrane curvature and completes the fission of a

12 COPII vesicle. Cell 122, 605-617.

13 Long, K.R., Yamamoto, Y., Baker, A.L., Watkins, S.C., Coyne, C.B., Conway, J.F., 14 and Aridor, M. (2010). Sar1 assembly regulates membrane constriction and ER export. 15 J. Cell Biol. 190, 115-128.

16 Ma, W., and Goldberg, J. (2016). TANGO1/cTAGE5 receptor as a polyvalent template 17 for assembly of large COPII coats. Proc. Natl. Acad. Sci. U. S. A. 113, 10061-10066.

18 Maeda, M., Katada, T., and Saito, K. (2017). TANGO1 recruits Sec16 to coordinately 19 organize ER exit sites for efficient secretion. J. Cell Biol. 216, 1731-1743.

20 Maeda, M., Saito, K., and Katada, T. (2016). Distinct isoform-specific complexes of 21 TANGO1 cooperatively facilitate collagen secretion from the endoplasmic reticulum. 22 Mol. Biol. Cell 27, 2688-2696.

23 Malhotra, V., and Erlmann, P. (2015). The pathway of collagen secretion. Annu. Rev.

24 Cell Dev. Biol. 31, 109-124.

25 Matsuoka, K., Orci, L., Amherdt, M., Bednarek, S.Y., Hamamoto, S., Schekman, R., 26 and Yeung, T. (1998). COPII-coated vesicle formation reconstituted with purified coat 27 proteins and chemically defined liposomes. Cell 93, 263-275.

28 McGourty, C.A., Akopian, D., Walsh, C., Gorur, A., Werner, A., Schekman, R., 29 Bautista, D., and Rape, M. (2016). Regulation of the CUL3 Ubiquitin Ligase by a 30 Calcium-Dependent Co-adaptor. Cell 167, 525-538 e514.

31 Miller, E.A., and Schekman, R. (2013). COPII - a flexible vesicle formation system. 32 Curr. Opin. Cell Biol.

33 Nakano, A., and Muramatsu, M. (1989). A novel GTP-binding protein, Sar1p, is 
1 involved in transport from the endoplasmic reticulum to the Golgi apparatus. J. Cell

2 Biol. 109, 2677-2691.

3 Nogueira, C., Erlmann, P., Villeneuve, J., Santos, A.J., Martinez-Alonso, E., 4 Martinez-Menarguez, J.A., and Malhotra, V. (2014). SLY1 and Syntaxin 18 specify a

5 distinct pathway for Procollagen VII export from the endoplasmic reticulum. Elife, $6 \mathrm{e} 02784$.

7 Raote, I., Ortega Bellido, M., Pirozzi, M., Zhang, C., Melville, D., Parashuraman, S.,

8 Zimmermann, T., and Malhotra, V. (2017). TANGO1 assembles into rings around

9 COPII coats at ER exit sites. J. Cell Biol. 216, 901-909.

10 Saito, K., Chen, M., Bard, F., Chen, S., Zhou, H., Woodley, D., Polischuk, R., 11 Schekman, R., and Malhotra, V. (2009). TANGO1 facilitates cargo loading at 12 endoplasmic reticulum exit sites. Cell 136, 891-902.

13 Saito, K., and Katada, T. (2015). Mechanisms for exporting large-sized cargoes from 14 the endoplasmic reticulum. Cell. Mol. Life Sci. 72, 3709-3720.

15 Saito, K., Maeda, M., and Katada, T. (2017). Regulation of the Sar1 GTPase Cycle Is 16 Necessary for Large Cargo Secretion from the Endoplasmic Reticulum. Front. Cell 17 Dev. Biol. 5, 75 .

18 Saito, K., Yamashiro, K., Ichikawa, Y., Erlmann, P., Kontani, K., Malhotra, V., and 19 Katada, T. (2011). cTAGE5 mediates collagen secretion through interaction with 20 TANGO1 at endoplasmic reticulum exit sites. Mol. Biol. Cell 22, 2301-2308.

21 Saito, K., Yamashiro, K., Shimazu, N., Tanabe, T., Kontani, K., and Katada, T. (2014).

22 Concentration of Sec12 at ER exit sites via interaction with cTAGE5 is required for 23 collagen export. J. Cell Biol. 206, 751-762.

24 Santos, A.J., Nogueira, C., Ortega-Bellido, M., and Malhotra, V. (2016). TANGO1 and 25 Mia2/cTAGE5 (TALI) cooperate to export bulky pre-chylomicrons/VLDLs from the 26 endoplasmic reticulum. J. Cell Biol. 213, 343-354.

27 Santos, A.J., Raote, I., Scarpa, M., Brouwers, N., and Malhotra, V. (2015). TANGO1 28 recruits ERGIC membranes to the endoplasmic reticulum for procollagen export. Elife $294, \mathrm{e} 10982$.

30 Sato, K., and Nakano, A. (2005). Dissection of COPII subunit-cargo assembly and 31 disassembly kinetics during Sar1p-GTP hydrolysis. Nat. Struct. Mol. Biol. 12, $32 \quad 167-174$.

33 Settles, E.I., Loftus, A.F., McKeown, A.N., and Parthasarathy, R. (2010). The vesicle 
1 trafficking protein Sar1 lowers lipid membrane rigidity. Biophys. J. 99, 1539-1545.

2 Tanabe, T., Maeda, M., Saito, K., and Katada, T. (2016). Dual function of cTAGE5 in

3 collagen export from the endoplasmic reticulum. Mol. Biol. Cell 27, 2008-2013.

4 Venditti, R., Scanu, T., Santoro, M., Di Tullio, G., Spaar, A., Gaibisso, R.,

5 Beznoussenko, G.V., Mironov, A.A., Mironov, A., Jr., Zelante, L., Piemontese, M.R.,

6 Notarangelo, A., Malhotra, V., Vertel, B.M., Wilson, C., and De Matteis, M.A. (2012).

7 Sedlin controls the ER export of procollagen by regulating the Sar1 cycle. Science 337,

$8 \quad 1668-1672$.

9 Wang, Y., Liu, L., Zhang, H., Fan, J., Zhang, F., Yu, M., Shi, L., Yang, L., Lam, S.M.,

10 Wang, H., Chen, X., Wang, Y., Gao, F., Shui, G., and Xu, Z. (2016). Mea6 controls

11 VLDL transport through the coordinated regulation of COPII assembly. Cell Res. 26, $12787-804$.

13 Yorimitsu, T., and Sato, K. (2012). Insights into structural and regulatory roles of

14 Sec16 in COPII vesicle formation at ER exit sites. Mol. Biol. Cell 23, 2930-2942.

15 Yoshihisa, T., Barlowe, C., and Schekman, R. (1993). Requirement for a

16 GTPase-activating protein in vesicle budding from the endoplasmic reticulum. Science $17 \quad 259,1466-1468$.

18 Zanetti, G., Prinz, S., Daum, S., Meister, A., Schekman, R., Bacia, K., and Briggs, J.A.

19 (2013). The structure of the COPII transport-vesicle coat assembled on membranes.

20 Elife 2, e00951.

21

22

23 


\section{FIGURE LEGENDS}

2 Figure 1. cTAGE5 enhances the GAP activity of Sec23 toward Sar1. (A) 200 nM Sar1 3 was incubated with a buffer consisting of $25 \mu \mathrm{g} / \mathrm{ml} \mathrm{Ni}$ liposome (20\%), $20 \mathrm{mM}$ 4 HEPES-KOH (pH 7.4), 100 mM KOAc, 6 mM Tris- $\mathrm{HCl}, 45 \mathrm{mM} \mathrm{NaCl}, 1 \mathrm{mM} \mathrm{MgCl}{ }_{2}$, $5 \quad 1.6 \%$ Glycerol, $133 \mathrm{nM}$ GDP, $2 \mu \mathrm{M}\left[\gamma^{-}{ }^{32} \mathrm{P}\right] \mathrm{GTP}, 50 \mu \mathrm{M}$ AppNHp, $67 \mu \mathrm{M} \beta$-ME, 67 $6 \mu \mathrm{M}$ AEBSF, in the presence or absence of $8 \mathrm{nM}$ FLAG-Sec12 (1-386 aa)-His ${ }_{6}, 15 \mathrm{nM}$ 7 HA-Sec23A/FLAG-Sec24D, $3.6 \mathrm{nM}$ cTAGE5 for $1 \mathrm{~h}$ at $30^{\circ} \mathrm{C}$. The amount of free ${ }^{32} \mathrm{Pi}$ 8 was quantified. (B) $200 \mathrm{nM}$ Sar1 was incubated with a buffer consisting of $25 \mu \mathrm{g} / \mathrm{ml}$ 9 liposome without Ni, 20 mM HEPES-KOH (pH 7.4), 100 mM KOAc, 6 mM Tris-HCl, $1045 \mathrm{mM} \mathrm{NaCl}, 1 \mathrm{mM} \mathrm{MgCl} 2,1.6 \%$ Glycerol, $133 \mathrm{nM}$ GDP, $2 \mu \mathrm{M}\left[\gamma-{ }^{32} \mathrm{P}\right] \mathrm{GTP}, 50 \mu \mathrm{M}$ 11 AppNHp, $67 \mu \mathrm{M} \beta$-ME, $67 \mu \mathrm{M}$ AEBSF, in the presence or absence of $8 \mathrm{nM}$ 12 FLAG-Sec12 (1-386 aa)-His 6 , 15 nM HA-Sec23A/FLAG-Sec24D and the indicated 13 concentration of cTAGE5 for $1 \mathrm{~h}$ at $30^{\circ} \mathrm{C}$. The amount of free ${ }^{32} \mathrm{Pi}$ was quantified. (C, 14 D) $200 \mathrm{nM}$ Sar1 was incubated with a buffer consisting of $25 \mu \mathrm{g} / \mathrm{ml} \mathrm{Ni}$ liposome 15 (20\%), 20 mM HEPES-KOH (pH 7.4), 100 mM KOAc, 6 mM Tris-HCl, 45 mM NaCl, $161 \mathrm{mM} \mathrm{MgCl} 2,1.6 \%$ Glycerol, $133 \mathrm{nM}$ GDP, $2 \mu \mathrm{M}\left[\gamma_{-}{ }^{32} \mathrm{P}\right] \mathrm{GTP}, 50 \mu \mathrm{M}$ AppNHp, 67 $17 \mu \mathrm{M} \beta$-ME, $67 \mu \mathrm{M}$ AEBSF, in the presence or absence of $8 \mathrm{nM}$ FLAG-Sec12 (1-386 18 aa)-His $_{6}, 15 \mathrm{nM}$ HA-Sec23A/FLAG-Sec24D and the indicated concentration of (C) 19 cTAGE5 or (D) Sec13/31 for $1 \mathrm{~h}$ at $30^{\circ} \mathrm{C}$. The amount of free ${ }^{32} \mathrm{Pi}$ was quantified.

21 Figure 2. Construction of cTAGE5 mutants lacking Sec23-binding activity. (A) 22 Purified FLAG-Sec23A or FLAG-Sec24D was immobilized onto FLAG agarose beads 23 and incubated with recombinant GST or GST-tagged cTAGE5-PRD (651-804aa)-His 6 24 or GST-tagged TANGO1-PRD (1651-1907 aa)-His ${ }_{6}$. Beads were washed and eluted 25 with FLAG peptide. Eluted proteins were subjected to SDS-PAGE followed by 26 western blotting with anti-FLAG and anti-GST antibodies. (B) PRD regions of 27 cTAGE5 mutants in pGADT7 plasmids were co-transformed with pGBKT7 plasmids 28 containing Sec23A into AH109 yeast strains and grown on tryptophan-, 29 leucine-deficient plate (-WL). Interactions were investigated by observing the cell 30 growth on tryptophan-, leucine-, histidine-, and adenine-deficient plate (-WLHA). (C) 31 Schematic representation of human cTAGE5 domain organization. sa, signal anchor; 32 TM, transmembrane; Coil, coiled-coil domain; PRD, proline-rich domain. The position 33 of R757 is shaded in yellow and the positions of 4PA mutations are shaded in light 
1 blue. (D) Recombinant GST or GST-tagged cTAGE5-PRD (651-804aa)-His 6 2 wild-type or cTAGE5-PRD R757G or cTAGE5-PRD 4PA were immobilized to 3 glutathione sepharose resin and incubated with FLAG-Sec23A. Resins were washed 4 and eluted with glutathione. Eluted proteins were subjected to SDS-PAGE followed by 5 western blotting with anti-FLAG and anti-GST antibodies. (E) Quantification of 6 Sec23A immunoblots $(n=9)$. The band intensities were normalized to those of GST 7 blots. Error bars represent mean \pm SEM. ${ }^{*} P P<0.001$. (F) HSC-1 cells were treated 8 with cTAGE5 siRNA and cultured for $24 \mathrm{~h}$. Then, FLAG-tagged cTAGE5 wild-type, 9 cTAGE5 R757G, or cTAGE5 4PA were transfected and further cultured for $24 \mathrm{~h}$. The 10 cells were fixed and stained with Sec16 and FLAG antibodies. Bars $=10 \mu \mathrm{m} .(\mathrm{G}, \mathrm{H})$ 11293 T cells were transfected with FLAG tag only, FLAG-tagged cTAGE5 wild-type, 12 cTAGE5 R757G, or cTAGE5 4PA with HA-tagged (G) TANGO1L or (H) Sec12. Cell 13 lysates were immunoprecipitated with anti-FLAG antibody and eluted with FLAG 14 peptide. Eluates and cell lysates were analyzed by SDS-PAGE followed by western blotting with FLAG or HA antibodies.

17 Figure 3. PRD of cTAGE5 enhances GAP acitivity of Sec23 toward Sar1. $200 \mathrm{nM}$ 18 Sar1 was incubated with a buffer consisting of $25 \mu \mathrm{g} / \mathrm{ml} \mathrm{Ni}$ liposome (10\%), $20 \mathrm{mM}$ 19 HEPES-KOH (pH 7.4), 100 mM KOAc, 8 mM Tris-HCl, $60 \mathrm{mM} \mathrm{NaCl}, 1 \mathrm{mM} \mathrm{MgCl}{ }_{2}$, $201.6 \%$ Glycerol, $133 \mathrm{nM}$ GDP, $2 \mu \mathrm{M}\left[\gamma^{-32} \mathrm{P}\right] \mathrm{GTP}, 50 \mu \mathrm{M}$ AppNHp, $67 \mu \mathrm{M} \beta$-ME, 67 $21 \mu \mathrm{M}$ AEBSF, 8 nM FLAG-Sec12 (1-386 aa)-His 6 , 15 nM HA-Sec23A/FLAG-Sec24D 22 and the indicated concentration of $\mathrm{His}_{6}$-GST, His ${ }_{6}$-cTAGE5 (651-804 aa)-GST 23 wild-type, $\mathrm{R} 757 \mathrm{G}, 4 \mathrm{PA}$ for $1 \mathrm{~h}$ at $30^{\circ} \mathrm{C}$. The amount of free ${ }^{32} \mathrm{Pi}$ was quantified.

Figure 4. cTAGE5 mutants with reduced Sec23-binding activity failed to secrete collagen VII. HSC-1 cells were treated with control or cTAGE5 siRNA and cultured 27 for $24 \mathrm{~h}$. For cTAGE5 siRNA-treated cells, FLAG-tagged cTAGE5 wild-type, 28 cTAGE5 R757G or cTAGE5 4PA were transfected and further cultured for $24 \mathrm{~h}$. The cells were fixed and stained with collagen VII and FLAG antibodies. Collagen VII 30 immunofluorescence signal per cell (arbitrary units, A.U.) were quantified in each cell 31 category described below. The cells positively stained with FLAG antibody were categorized as the constructs expressed, and the surrounding unstained cells were categorized as non-transfected counterparts. Within each well, cells transfected with 
1 constructs are labeled as + and non-transfected cells are labeled as -. Analysis of

2 variance. Error bars represent mean \pm SEM; $* * P<0.001$; n.s., $P>0.05$. The data

3 shown are from single representative experiment out of four replicates. Cells treated 4 with control siRNA $(n=65)$; cells treated with cTAGE5 siRNA and wild-type- $(n=$ $5 \quad 162)$; wild-type $+(n=53) ; \mathrm{R} 757 \mathrm{G}-(n=151) ; \mathrm{R} 757 \mathrm{G}+(n=53) ; 4 \mathrm{PA}-(n=177)$; $6 \quad 4 \mathrm{PA}+(n=57)$.

8 Supplemental Figure 1. Recombinant human proteins used in the GTPase hydrolysis 9 assay were resolved by SDS-PAGE followed by CBB staining. (A) $1.0 \mu \mathrm{g}$ Sar1A. (B) $10 \quad 0.6 \mu \mathrm{g}$ FLAG-Sec12 (1-386 aa)-His ${ }_{6}$ (lane 1), $0.43 \mu \mathrm{g}$ HA-Sec23A/0.6 $\mu \mathrm{g}$ 11 FLAG-Sec24D (lane 2), $0.54 \mu \mathrm{g}$ Sec13/2.0 $\mu \mathrm{g}$ FLAG-Sec31A (lane 3). (C) $10 \mathrm{ng}$ $12 \mathrm{His}_{6}$-cTAGE5 (61-804 aa)-FLAG. (D) $2.6 \mu \mathrm{g} \mathrm{His}_{6}$-GST, $2.25 \mu \mathrm{g} \mathrm{His}_{6}$-cTAGE5 (PRD) WT, RG, 4PA.

Supplemental Figure 2. 200 nM Sar1 and 15 nM HA-Sec23A/FLAG-Sec24D were incubated with a buffer consisting $25 \mu \mathrm{g} / \mathrm{ml} \mathrm{Ni}$ liposome (20\%), $20 \mathrm{mM}$ HEPES-KOH (pH 7.4), 100 mM KOAc, 6 mM Tris- $\mathrm{HCl}, 45 \mathrm{mM} \mathrm{NaCl}, 1 \mathrm{mM} \mathrm{MgCl}{ }_{2}, 1.6 \%$ Glycerol, $133 \mathrm{nM}$ GDP, $2 \mu \mathrm{M}\left[\gamma^{-32} \mathrm{P}\right] \mathrm{GTP}, 50 \mu \mathrm{M}$ AppNHp, $67 \mu \mathrm{M} \beta$-ME, $67 \mu \mathrm{M}$ AEBSF, in the presence or absence of $8 \mathrm{nM}$ FLAG-Sec12 (1-386 aa)-His ${ }_{6}$, and $3.6 \mathrm{nM}$ cTAGE5 for $1 \mathrm{~h}$ at $30^{\circ} \mathrm{C}$. The amount of free ${ }^{32} \mathrm{Pi}$ was quantified, $n=6 .{ }^{*} \mathrm{p}<0.05$. Error bars represent means \pm SEM.

Supplemental Figure 3. $200 \mathrm{nM}$ Sar1 was incubated with a buffer consisting 25 $\mu \mathrm{g} / \mathrm{ml} \mathrm{Ni} \mathrm{liposome} \mathrm{(10 \% ),} 20$ mM HEPES-KOH (pH 7.4), $100 \mathrm{mM} \mathrm{KOAc,} 8 \mathrm{mM}$ Tris- $\mathrm{HCl}, 60 \mathrm{mM} \mathrm{NaCl}, 1 \mathrm{mM} \mathrm{MgCl}{ }_{2}, 1.6 \%$ Glycerol, $133 \mathrm{nM}$ GDP, $2 \mu \mathrm{M}\left[\gamma^{-32} \mathrm{P}\right]$ GTP, $50 \mu \mathrm{M}$ AppNHp, $67 \mu \mathrm{M} \beta$-ME, $67 \mu \mathrm{M}$ AEBSF, 8 nM FLAG-Sec12 (1-386 aa)-His ${ }_{6}, 15 \mathrm{nM} \mathrm{HA}-\mathrm{Sec} 23 \mathrm{~A} / \mathrm{FLAG}-\mathrm{Sec} 24 \mathrm{D}$ and $400 \mathrm{nM}$ (A) or $800 \mathrm{nM}$ (B) of

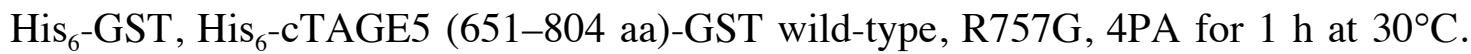

29 The amount of free ${ }^{32} \mathrm{Pi}$ was quantified, $n=5$. ${ }^{*} \mathrm{p}<0.05$. n.s., not significant compared 30 with $\mathrm{His}_{6}$-GST. Error bars represent means \pm SEM. 
A

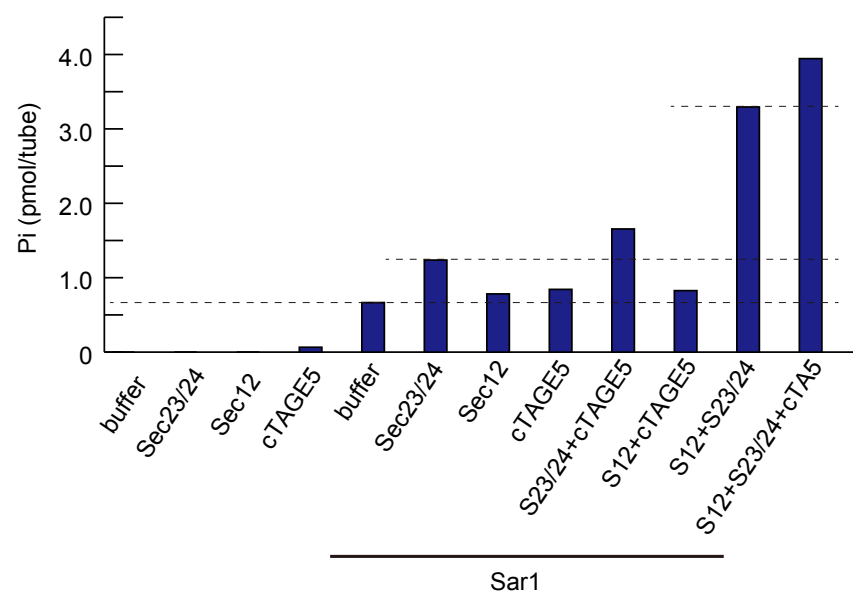

C

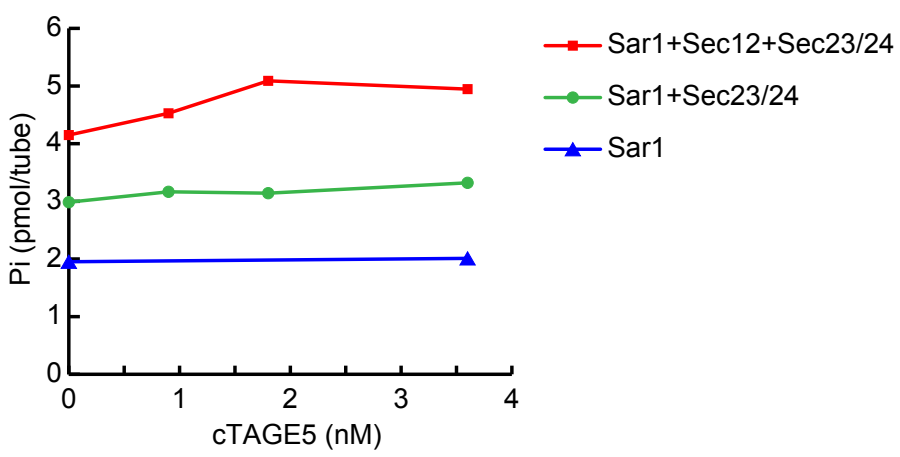

B

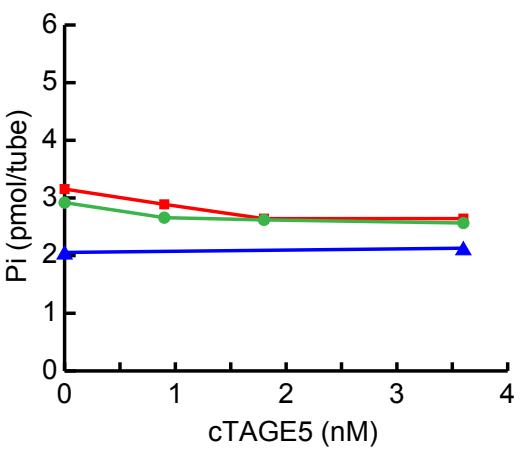

$\longrightarrow$ Sar1+Sec12+Sec23/24

$\longrightarrow$ Sar1+Sec23/24

$\dashv$ Sar1
D

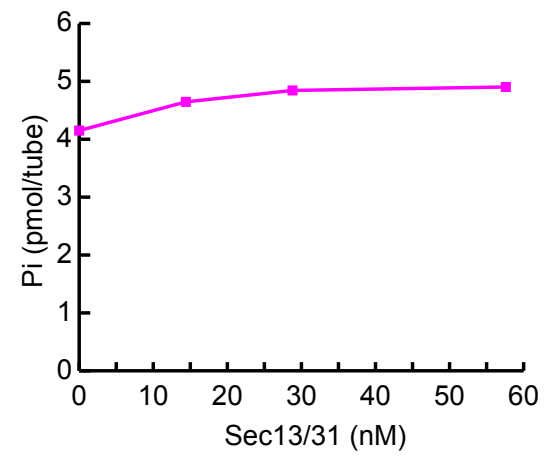

Fig. 1 
A

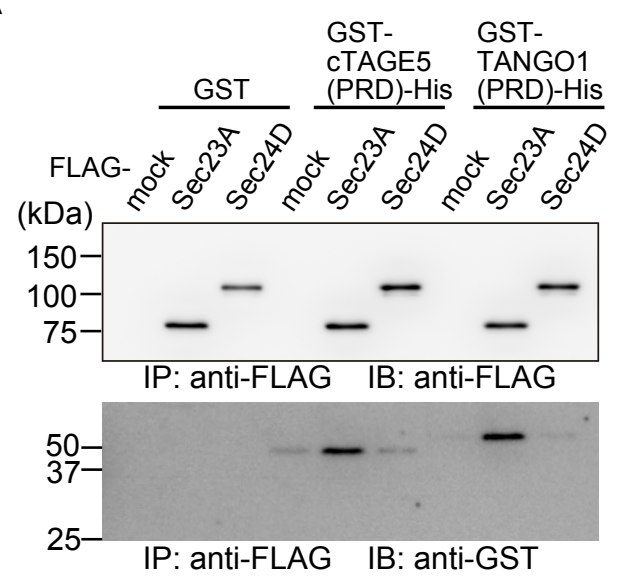

B

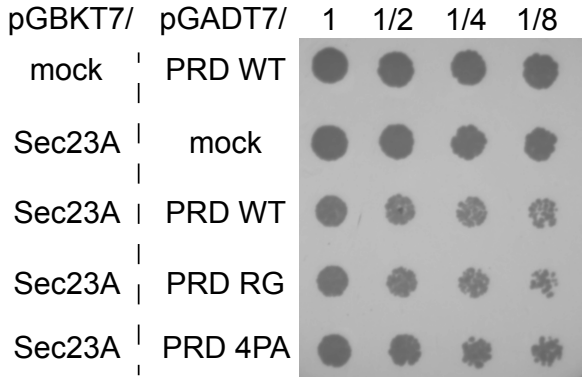

$-W L$

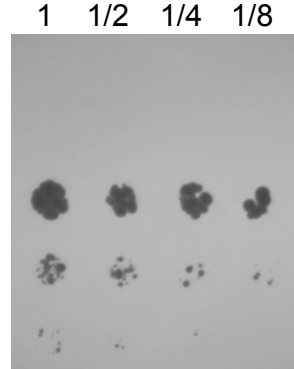

-WLHA

C

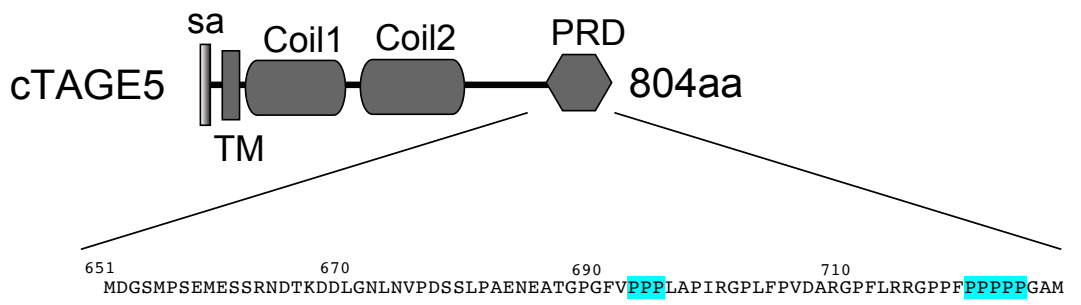

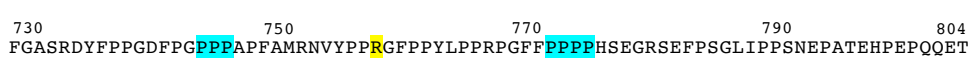

$\mathrm{D}$

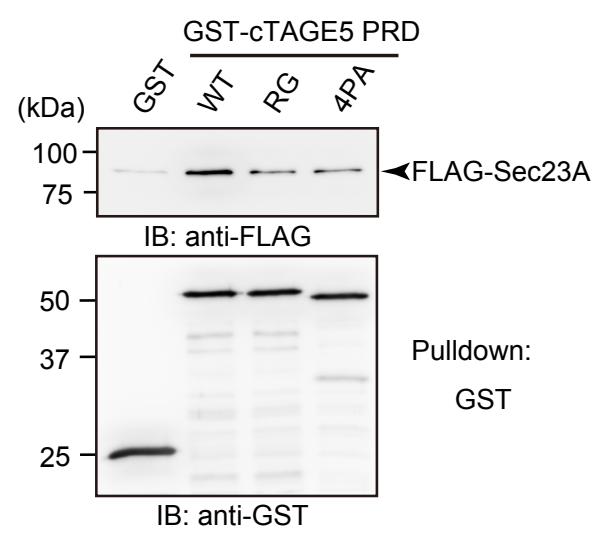

G
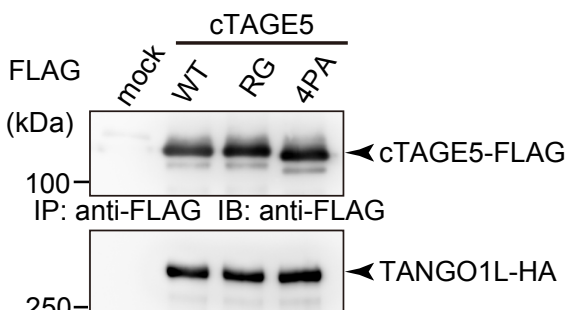

IP: anti-FLAG IB: anti-HA 250 $-\infty-\infty$ TANGO1L-HA
$E$

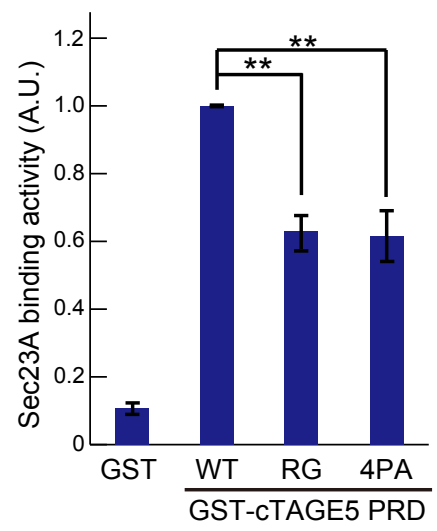

$\mathrm{H}$
F

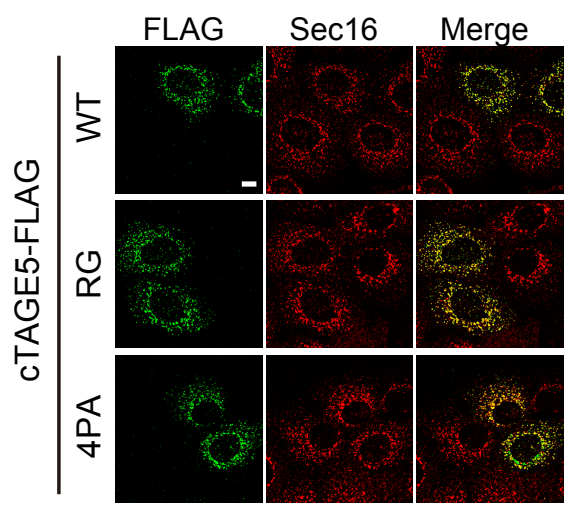


Sar1+Sec12+Sec23/24+

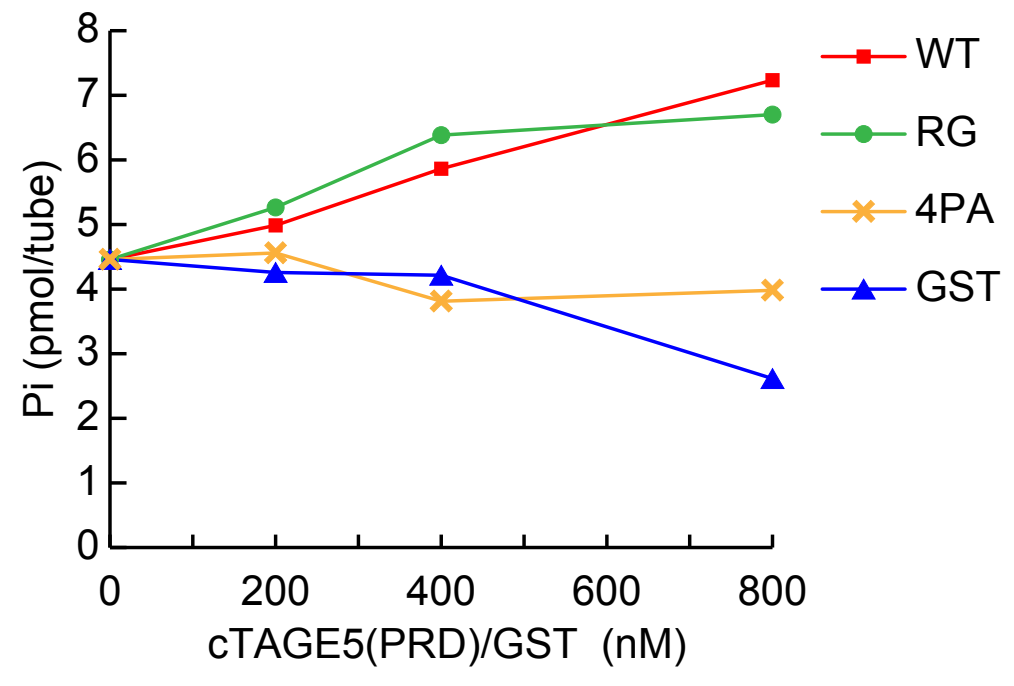

Fig. 3 


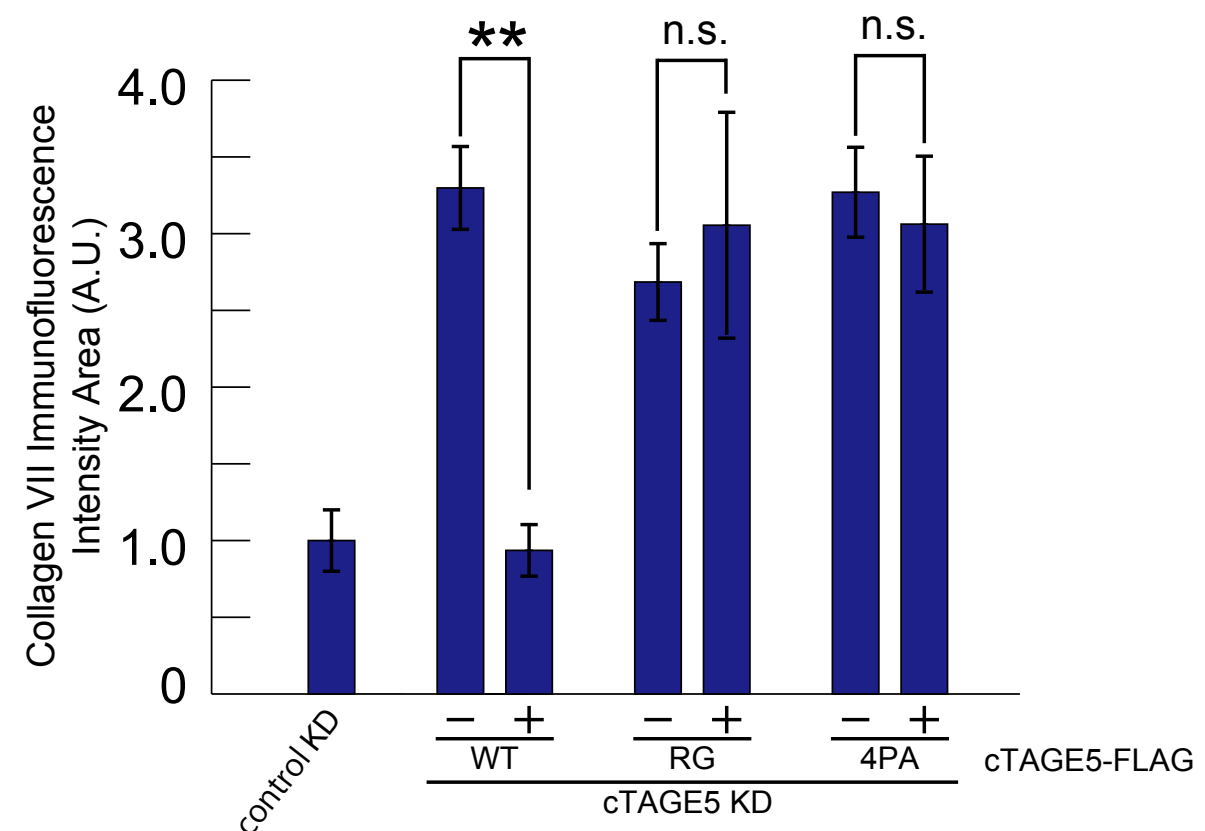

Fig. 4 
A

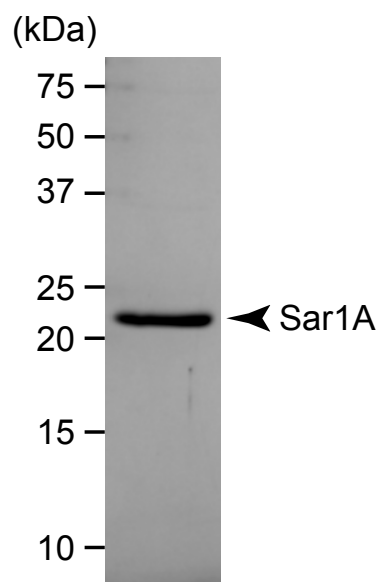

B

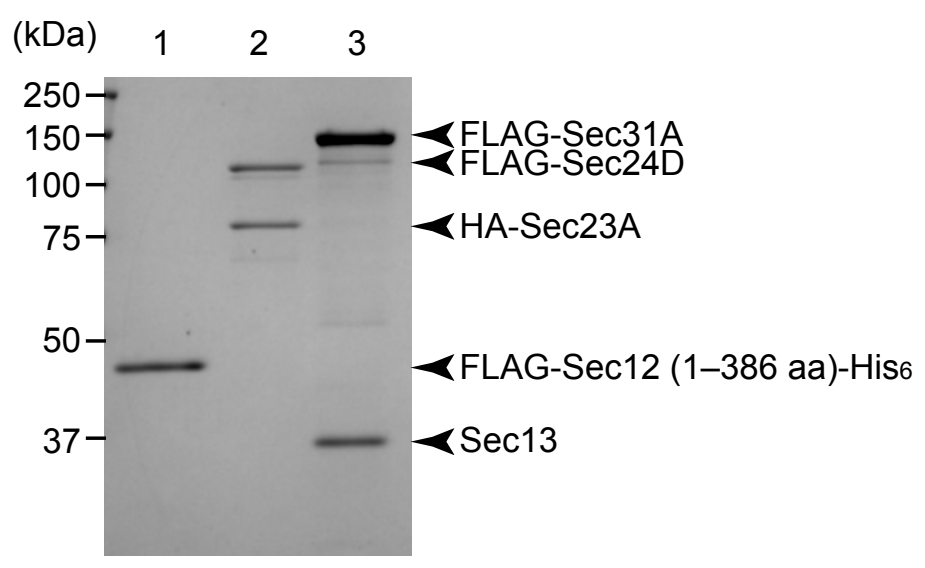

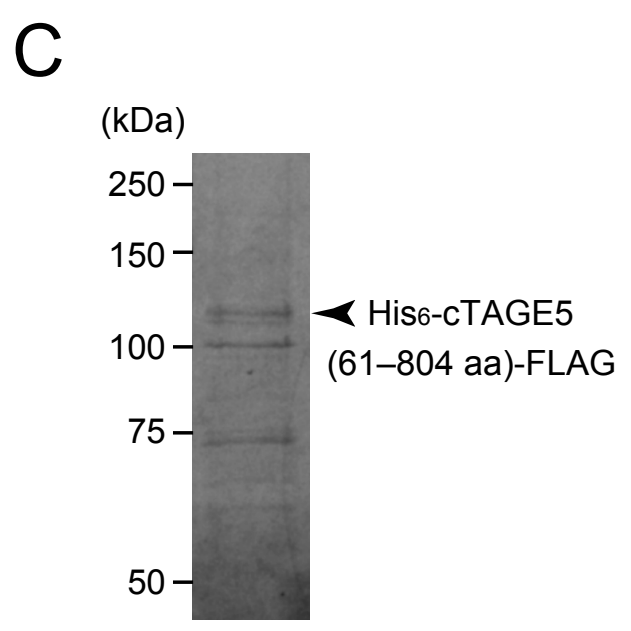

D

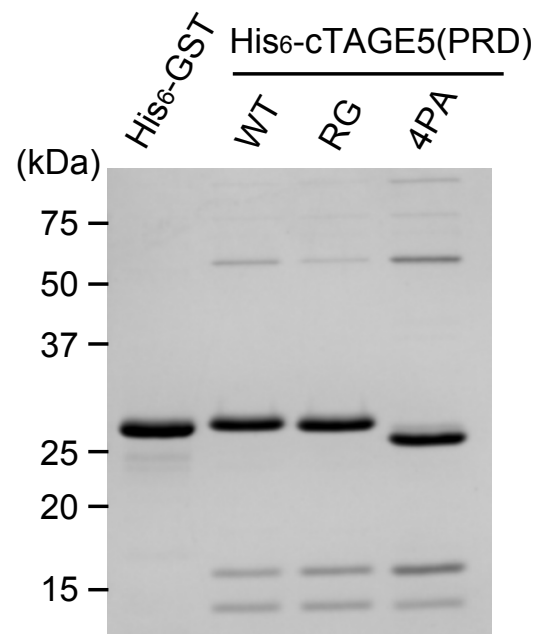

Fig. S1 


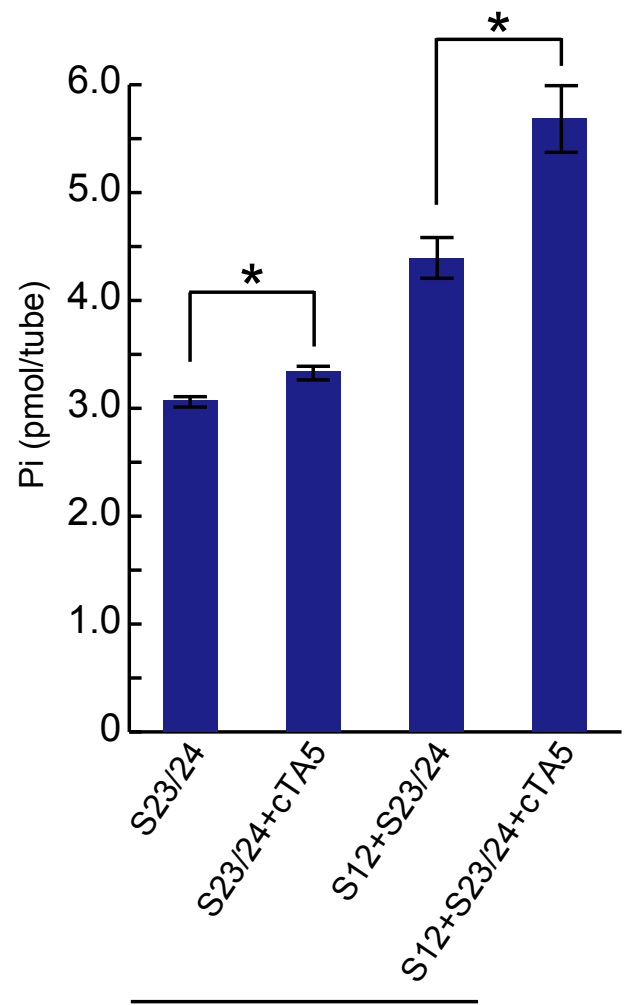

Sar1

Fig. S2 
A

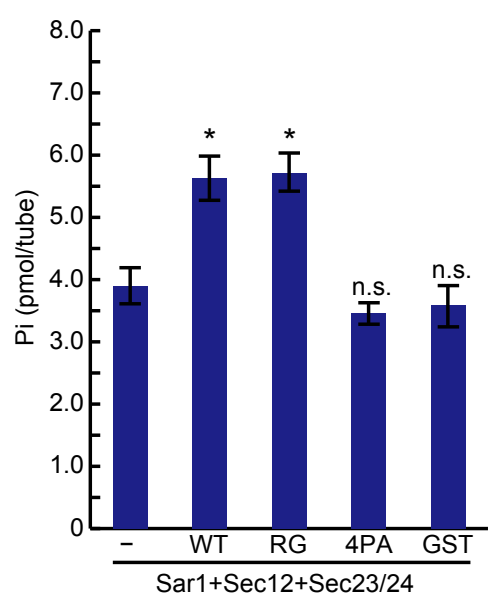

B

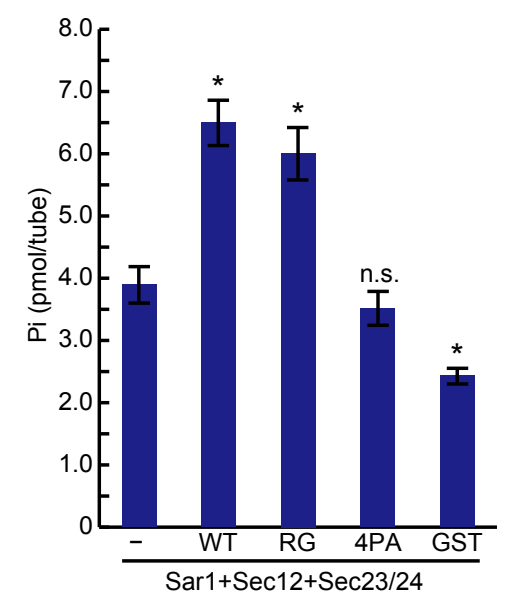

Fig. S3 